\title{
Exploration of Virtual Simulation Experiment Teaching of Network Communication Courses Based on Cloud Computing
}

\author{
Hui Wang ${ }^{1, *}$, Zhipeng $\mathrm{Si}^{1}$, and Bo Hong ${ }^{1}$ \\ ${ }^{1}$ School of Computer Science and Engineering, Xi' an Technological University, Xi'an 710021, China \\ *Corresponding author. Email: wanghuihy@xatu.edu.cn
}

\begin{abstract}
The novel coronavirus pneumonia has changed colleges and universities' traditional classroom teaching mode, especially when the current epidemic situation is still severe; the teaching mode and teaching content need to be adjusted in time. Aiming at the deficiencies in the experimental teaching of network communication courses, this thesis carried out practical teaching exploration of virtual simulation experiments of network communication courses based on cloud computing combined with the actual needs of practical network talents, and constructed a hierarchical virtual simulation experimental teaching system. The established virtual simulation experiment platform and teaching system provide better support for the development of experimental teaching of network communication courses, and have achieved better implementation results. Keywords: network communication courses; cloud computing; virtual simulation experiment; experimental
\end{abstract} teaching system; teaching platform of virtual simulation experiment

\section{INTRODUCTION}

The novel coronavirus pneumonia has changed the traditional classroom teaching mode in colleges and universities, especially in the current situation of the epidemic is still grim, so it is necessary to adjust the teaching mode and teaching content in time. Moreover, compared with theoretical courses, practical courses have fewer online teaching resources, and the teaching effect of live broadcasting is not ideal[1]. However, practice link plays a pivotal role in the undergraduate teaching system, which is the foundation of engineering major and an important way to improve students' practical ability, practical ability and talent cultivation quality. In engineering certification, it is clearly required to continuously improve the experimental teaching system. In the face of the global long-term continuous novel coronavirus pneumonia(NCL), aiming at the shortcomings of network communication course experimental teaching and combining with the actual needs of network practical talents, this paper carries out the exploration and reform of the practical teaching of virtual simulation experiment of network communication course based on cloud computing[2]. Through careful study, the internal relationship between experimental courses is excavated, and the course experiments of network communication courses are effectively integrated, and a hierarchical virtual simulation experimental teaching system is constructed[3].

\section{CURRENT SITUATION AND PROBLEMS}

Experimental teaching based on virtual simulation technology is an important content of information construction in higher education. At the same time, it is also the product of deep integration of subject specialty and information technology, and it is an effective supplement to traditional experimental teaching. In recent years, with the rapid development of computer network, electronics, communication and other technologies, many institutions at home and abroad have carried out research on virtual experiments[4].

Network communication courses are practical courses, and experimental teaching plays a significant role in professional education and teaching. Moreover, as the proportion of virtual simulation experiment in experimental teaching increases gradually, its influence on the overall teaching quality is also increasing[5]. The School of Computer Science at Xi'an Technological University has been building virtual simulation experiments since 2018 to make up for the shortage of hardware equipment in our school. At present, the completed virtual simulation experiment projects include VLAN division and communication between VLANs, virtual simulation experiment of urban intelligent transportation and virtual simulation experiment of network security. These experiments are respectively the in-class experiments of "Computer Communication and Network", "Innovative Design of Internet of Things System" and "Introduction to Network Security Technology" and other courses. 
However, with the development of virtual simulation experiment projects and the continuation of the original experiment teaching mode, the practical teaching of network communication courses has the following problems[6].

1) The existing laboratory hardware equipment has been unable to meet the increasing needs of experimental teaching

2) It is not effective to integrate each isolated virtual simulation experiment project, which is not conducive to the cultivation of students' comprehensive practice ability.

3) The existing practice teaching operation effect is not ideal, in-depth analysis of the experimental effect and continuous improvement of the strength is insufficient.

\section{VIRTUAL SIMULATION EXPERIMENT TEACHING PLATFORM BASED ON CLOUD COMPUTING}

The design of virtual simulation experimental teaching platform is an effective support for network communication courses to provide virtual simulation experimental teaching. The platform can expand experimental projects according to the change of teaching tasks, and the teaching content can cover different levels of software and hardware, so as to open multiple courses under the same platform environment. The whole platform is designed into four levels: cloud computing platform, virtual simulation platform, virtual simulation experiment and remote access interface.

\subsection{Cloud Computing Platform}

Cloud computing is an enhancement pattern of internetbased related services, which usually refers to the provision of dynamic, easily scalable and often virtualized resources over the Internet. The "cloud" of cloud computing is the collection of resources existing in the server cluster in the computer network. It includes not only the hardware equipment such as tower or blade server, storage magnetic array, switch, router, but also the software resources such as server operating system, management software, virtual machine operating system, application software and so on. Cloud computing platform is the foundation of the whole virtual simulation experiment teaching platform, all experiments and data management all rely on the virtual enhancement services provided by cloud computing.

\subsection{Virtual Simulation Platform}

Virtual simulation experimental platform of network communication courses is the core platform of simulation operation, including experimental teaching resource management, experimental teaching management, experimental teaching administration management, online attendance checking, online testing, auxiliary question- answering and other systems. These systems are for the virtual simulation experiment to do auxiliary work, and the management and simulation of these systems rely on the virtual simulation platform.

\subsection{Virtual Simulation Experiment}

With the support of the virtual simulation platform, we can realize the virtual simulation experiment which has been designed. Through the analysis and construction of virtual simulation experiment system of network communication courses, we divide the simulation experiment of network communication courses into four modules, which are basic experiment, comprehensive experiment, research experiment and application innovation experiment. Among them, the basic experimental system can complete RFID, sensors and other basic skills experiments. The comprehensive experiment can complete the comprehensive application development based on the upper application system of Android, C\# and Java and the lower hardware communication. Research experiments can be combined with scientific research projects to complete extracurricular experiments to achieve practical exploration. Modules such as smart agriculture, smart home, smart books, 3D virtual simulation experimental teaching system, and smart mall set up a typical practice environment for the comprehensive application and innovation system of the Internet of Things, which can help students experience the actual application scenarios of the Internet of Things.

\subsection{The Interface for Remote Access}

The top layer provides the remote access interface of various intelligent devices (PC, laptop, smart phone, etc.) to realize the access anytime and anywhere.

The architecture of virtual simulation experimental teaching platform for network communication courses that integrates virtual simulation technology and cloud computing is shown in Figure 1.

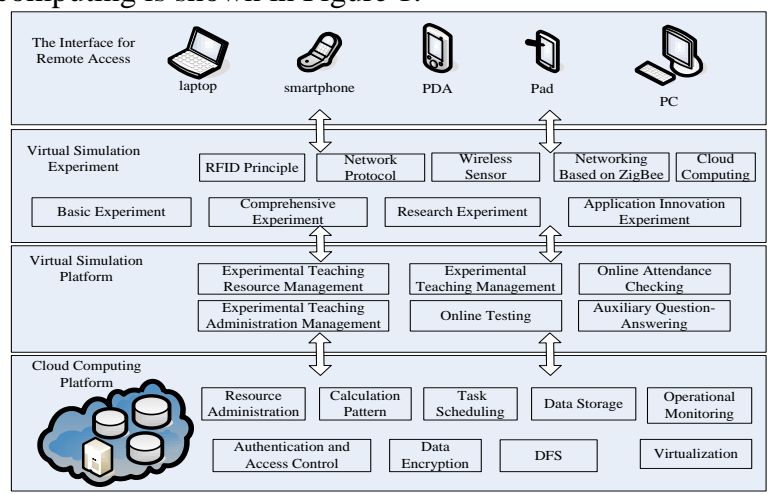

Figure 1. The architecture of virtual simulation experimental teaching platform for network communication courses 
The virtual simulation experimental teaching platform takes the cultivation of students' ability as the main line, and its core is to cultivate students' comprehensive ability to flexibly use theoretical knowledge, combine knowledge with skills and solve network engineering problems.

When designing the virtual simulation experimental platform of network communication courses, it is necessary for us to consider the use of existing hardware resources such as virtual simulation software and experimental box. If the construction of the virtual simulation experimental teaching platform is completely different from the existing physical system, it is bound to make the experimental teachers have to change to the virtual simulation experimental platform to carry out experimental teaching, which will greatly increase the workload and training time of the experimental teachers. Therefore, the existing hardware and software resources and teachers' skills should be taken into consideration when building the virtual simulation experimental platform in the later stage.

Virtual simulation experiment teaching platform is implemented as shown in Figure 2, the framework of the platform provides a comprehensive range of virtual experimental teaching auxiliary functions, including: intelligent directions of experiment teaching management, teaching process, corrects the results automatically, experiment report, system management, and other functions, provide service for virtual experiment teaching environment and the corresponding application. The experimental teaching platform sets up three types of roles: system administrator, teacher and student. The user name of the student is set as the student number by default, and the user name of the teacher is set as the teacher number by default.

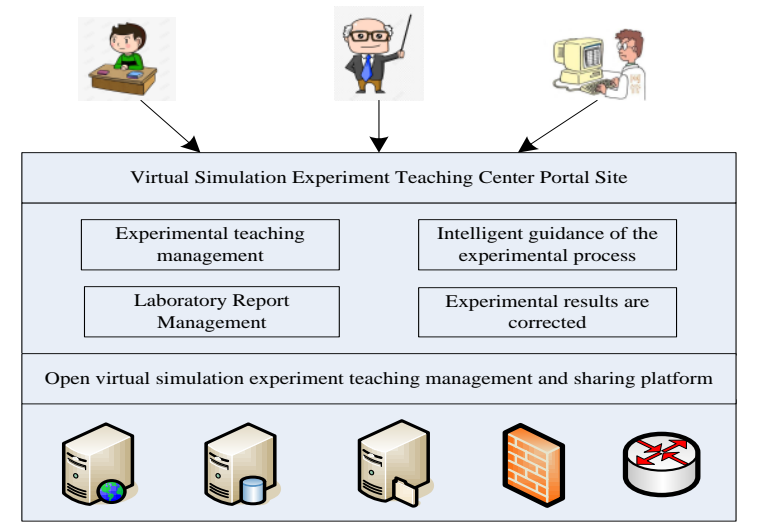

Figure 2. Function frame diagram of virtual simulation experiment teaching platform

At present, the platform has three courses including VLAN partition and VLAN communication, virtual simulation experiment of urban intelligent transportation and virtual simulation experiment of network security. $\log$ in the system as a teacher and see the teaching navigation diagram. Through the design and deployment of the virtual simulation experimental platform, not only the coupling degree between the teaching and experimental system is closer, but also the problem of "information island" can be solved.

\section{CONSTRUCTING THE VIRTUAL SIMULATION EXPERIMENT TEACHING SYSTEM OF NETWORK COMMUNICATION COURSES}

In view of the needs of computer network communication talents training, the core knowledge points of computer network, wireless network, single-chip computer, embedded development, RFID tag design and application and other professional courses are studied.

According to the basic experiment, design experiment and comprehensive experiment of related courses, a systematic and scientific virtual simulation experiment teaching system of network communication courses is constructed to promote the reform of experimental teaching of related courses, which is helpful to improve the overall level of experimental teaching of network communication courses.

\subsection{The Goal for Virtual Simulation Experiment Teaching System}

Network communication based on cloud computing courses experimental teaching system of virtual simulation of the main construction goal is through the study of virtual experiment environment and high simulation experiment object, build a scientific and systematic virtual simulation experiment teaching system, and use the virtual simulation experiment platform, lets the student in the virtual experiment teaching environment to carry out the network communications suite cannot conduct or difficult to complete the experiment, to complete physical teaching experiment teaching outline the requirements of the teaching effect. Therefore, in the network communication classes virtual simulation in the process of the reform of experimental teaching system, through the investigation and research has approved national or provincial network communication courses in colleges of virtual simulation experimental teaching situation, combined with the actual situation of the network communication classes in our school, by adopting the combination of theory and practice research methods, focus on research, while using, while teaching, practice, combining the teaching practice. In addition, virtual simulation experiments of network communication courses should highlight the guiding ideology of "scientific planning and resource sharing". With the help of virtual reality, network communication, intelligent science and human-computer interaction and other technologies, remote and networked experimental teaching can be realized, so that students can carry out experiments anytime and anywhere. After certain conditions are met, it is also possible to combine network communication technology with cloud computing, big data analysis, artificial intelligence, etc., and make full use of cloud computing technology to provide students with conditions for conducting experiments remotely through the network anytime and anywhere. 


\subsection{The Core Knowledge Points and Their Relations}

Through the analysis and research of relevant courses, we obtained the relationship among the core knowledge points of network communication courses, as shown in Figure 3.

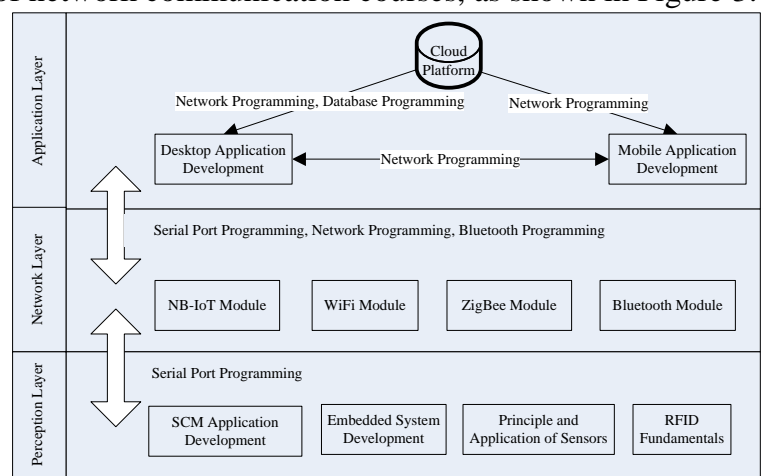

Figure 3. The core knowledge points of network communication courses

Knowledge points of network communication courses cover hardware and software. How to effectively integrate software experiments with hardware virtual simulation experiments is one of the main contents of this research.

The core knowledge points of network communication courses are studied from three levels: perception layer, network layer and application layer.

\subsection{Hierarchy System of Virtual Simulation Experiment Teaching}

First of all, based on the analysis and research of the core knowledge points of network communication courses, this paper proposes the idea of integrating theoretical foundation and applied technology from four levels, namely, perception layer, network layer, security layer and application layer. Secondly, the course is assigned to a corresponding level, the design of the basic experimental projects and comprehensive experimental projects related to the course, and these experimental projects through serial communication programming, network communication and network protocol analysis and other professional knowledge, better solve the application and development of network communication system. Finally, the teaching system of virtual simulation experiment is designed according to the hierarchical structure, the teaching resources are integrated, and the resources are centrally managed and allocated to maximize the effectiveness of the virtual simulation experiment course. Combined with the course content of network communication courses, a systematic and scientific teaching system architecture of virtual simulation experiment of network communication courses is constructed based on hierarchical analysis method, as shown in Figure 4.

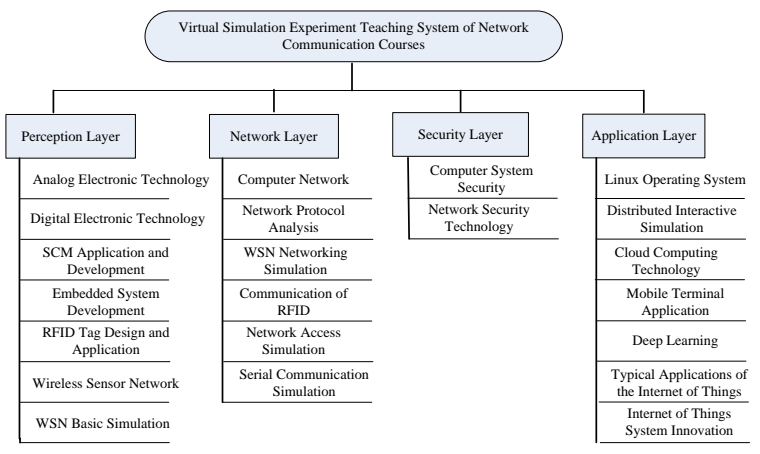

Figure 4. Virtual simulation experiment teaching system of network communication courses

Through the further research and exploration of virtual simulation experiment teaching, the experiment curriculum system, the experiment content and the experiment teaching method are optimized and reformed comprehensively. This is an important way to improve the practical ability of students in network communication courses, and plays a certain guiding role in the training of talents in network communication courses. The virtual simulation experiment project built through serial communication programming, network communication programming and database programming and other professional knowledge, better solve the problems in the design and implementation of network communication application system. In addition, the introduction of modern frontier hot technology into the experimental teaching content is of special social significance for the implementation of the frontier and engineering teaching activities.

\section{IMPLEMENTATION EFFECT}

Virtual simulation experimental teaching platform based on cloud computing can facilitate teachers to bring experimental projects into the classroom. In the severe situation of the epidemic, virtual simulation experiments can be effectively used to carry out on-site operation and demonstration in class, making theoretical knowledge more vivid and vivid. At the same time, it also solved the experimental classes cannot meet the problem of students complete the experiment, the network communication courses related experiment course is no longer confined to theoretical classroom and laboratory, on the teaching time and space to expand the student to the network communication courses learning scope of relevant experimental course, for the experimental teaching of computer related professional will provide better support.

\subsection{Unified Simulation Teaching Platform Reduces the Teaching Cost}

To some extent, the virtual simulation experimental teaching platform based on cloud computing can effectively solve the shortage of funds, insufficient investment in equipment, delayed equipment update, 
equipment aging, large-scale experimental environment cannot be simulated and other situations. To a certain extent, it alleviates the link that cannot be realized in real laboratory teaching in space and time, and effectively reduces the cost of experimental teaching.

\subsection{The Perfect Experimental Teaching System Effectively Integrates Teaching Resources}

With the help of virtual simulation technology and simulation software, it simulates the real working environment, constructs an effective virtual simulation teaching mode, improves the quality of practical teaching resources in terms of innovation and advanced nature, and enables the experimental teaching resources to meet the special experiments that the real experimental platform cannot carry out.

\subsection{The Rich and Varied Teaching Modes Improve the Quality of Practical Teaching}

The traditional experimental teaching mode is in the laboratory, the teacher speaks, the student listens, the form is very single. In the virtual simulation platform based on the construction of the network communication classes virtual simulation experiment teaching system. In addition, it is of great help and practical significance to transform teachers' existing scientific research achievements into virtual simulation experiment contents, enrich experimental teaching, expand students' ideas and explore students' experiments, which can improve the quality of applied talents of network communication and enhance students' innovative scientific research consciousness and innovation ability. The combination of "traditional experiment teaching + virtual simulation experiment teaching" is used to improve the quality of experiment teaching.

\section{CONCLUSION}

Explore and reform the virtual simulation experimental teaching system of network communication courses based on cloud computing, and reform and improve the existing experimental teaching system of network communication courses. Through the effective research and exploration of virtual simulation experiment teaching, the internal connection between the experimental courses is excavated, and the network communication course experiment is effectively integrated.

The construction of a good virtual simulation experimental platform and teaching system can provide better support for the development of experimental teaching of network communication courses. On the one hand, after the reform, the network communication courses have become more mature in terms of teaching staff and practical teaching. The practical contents impart can reflect the latest network communication technology and close to the actual social needs, so that students can apply what they have learned and achieve achievements. On the other hand, in the process of teaching and learning, teachers and students can realize communication and interaction, and promote teachers themselves to grasp the cutting-edge technology, so that teaching and learning can benefit each other. In the future, based on the achievements of the reform, we will explore the promotion and use of the existing virtual simulation experimental resources in other majors and colleges, further improve the utilization rate of virtual resources, and promote the cultivation of complex, innovative and outstanding talents.

\section{ACKNOWLEDGMENT}

This research was financially supported by the key research and development plan project of Shaanxi Science and Technology Department (Grant No. 2017ZDXM-GY-016), and the project of Innovation and Entrepreneurship Training Program for College Students at the national level (Grant No. S202010702112), and the Research project on teaching reform of education in Shaanxi province (Grant No. 20JGY016, 20JGZ007).

\section{REFERENCES}

[1]L Yafeng, Y Longjiang, L qunwei, S Li and W Yuanxi, "IoT virtual simulation experiment teaching platform - network and embedded gateway layer," Research and Exploration in Laboratory, 2019, 38 (12):122-126.

[2]C Shi, L Yuehua, C Xiaoyong, C Shanli and G ye, "Construction of virtual simulation experiment platform with cloud computing architecture," Research and Exploration in Laboratory, 2020,39(12):238-241.

[3]Z Liya, Y Maode and G Limin "Teaching Reform and Practice of Electrical and Electronic Experiment

Based on Virtual Simulation," Experiment Science and Technology, 2018, 16(2):129-133.

[4]O Y Zhiyou, Y Chunlei, “Construction and research of virtual simulation experiment teaching platform for internet of things," Computer Education, 2017(12):155-158.

[5]L Yafeng, Y Longjiang, L qunwei, S Li and W Yuanxi, "The construction scheme of virtual simulation teaching resource under the background of education informatization," Experiment Science and Technology, 2018(2):195-198.

[6]Z Ying, C Yanfei, G Kai and S Jinsong, "Exploration of the virtual simulation online teaching of practical course during the COVID-19 outbreak," Research and Exploration in Laboratory, 2020,39(11):119-122+128. 